\title{
Surface state atoms and their contribution to the surface tension of quantum liquids
}

\author{
A. D. Grigoriev \\ Samara State University, Samara, Russia \\ A. M. Dyugaev ${ }^{*}$ and P. D. Grigoriev \\ L. D. Landau Institute for Theoretical Physics, Chernogolovka, Russia
}

\begin{abstract}
We investigate the new type of excitations on the surface of liquid helium. These excitations, called surfons, appear because helium atoms have discrete energy level at the liquid surface, being attracted to the surface by the van der Waals force and repulsed at a hard-core interatomic distance. The concentration of the surfons increases with temperature. The surfons propagate along the surface and form a two-dimensional gas. Basing on the simple model of the surfon microscopic structure, we estimate the surfon activation energy and effective mass for both helium isotopes. We also calculate the contribution of the surfons to the temperature dependence of the surface tension. This contribution explains the great and long-standing discrepancy between theory and experiment on this temperature dependence in both helium isotopes. The achieved agreement between our theory and experiment is extremely high. The comparison with experiment allows to extract the surfon activation energy and effective mass. The values of these surfon microscopic parameters are in a reasonable agreement with the calculated from the proposed simple model of surfon structure.
\end{abstract}

PACS numbers: 73.20.Dx,67.55.S

\section{INTRODUCTION}

The microscopic description of the surface of liquids touches various fields of natural science. This problem is not simple, and even the calculation of the surface tension coefficient has many difficulties. $\frac{1}{}$ At low temperature the quantum nature of the surface excitations becomes important. At very low temperature only few types of surface excitations with the energy less or of the order of temperature are relevant for the problem. This fact greatly simplifies the description of liquid surface in the lowtemperature limit. An accurate calculation of the absolute value of the surface tension coefficient $\alpha$ remains a challenge, but the calculation of its temperature dependence $\alpha(T)$ is much simpler. Among all liquids, the low-temperature limit is reached only in helium, and the surface of liquid helium has been studied experimentally in great detail $\stackrel{2}{2}$ Therefore, we apply our analysis mainly to the liquid helium. The experimental values of the surface tension of liquid ${ }^{4} \mathrm{He}$ and ${ }^{3} \mathrm{He}$ at zero temperature $\operatorname{are}^{3,4} \alpha_{H e 4}(T=0) \equiv \alpha_{04}=0.3544 \mathrm{dyn} / \mathrm{cm}$ and $\alpha_{H e 3}(T=0) \equiv \alpha_{03}=0.155 \mathrm{dyn} / \mathrm{cm}$. Taking these values as a reference point, one may calculate the deviation $\Delta \alpha(T) \equiv \alpha_{0}-\alpha(T)$ as the sum of the contributions from all types of surface excitations to the surface free energy per unit square. At low temperature the concentration of these excitations is low, and the interaction between the excitations can be neglected. Then the surface excitations form an ideal two-dimensional gas of particles with the dispersion determined by the nature of these excitations. The free energy of this gas is well known ${ }^{5}$ [see Eq. (19) below].

At sufficiently low temperatures, the only considered type of surface excitations are the quanta of surface waves, called ripplons. The ripplons lead to the temperature dependence of the surface tension of a liquid given by the Atkins formula ${ }^{6}$ :

$$
\Delta \alpha_{R}(T)=A T^{7 / 3}
$$

where the coefficient $A=6.8 \mathrm{mdyn} / \mathrm{cm} \cdot \mathrm{K}^{7 / 3}$ for ${ }^{4} \mathrm{He}$. However, this estimate of the temperature dependence of the surface tension is much weaker than the measured one.$^{\frac{3}{3}}$ Moreover, Eq. (1) applies only for ${ }^{4} \mathrm{He}$ in the superfluid state, because above the $\lambda$-point, $T_{\lambda}=2.17 \mathrm{~K}$, the short-wavelength ripplons are damped by the liquid viscosity. The damping of the high-energy short-wavelength ripplons must lead to the strong modification of the dependence (1) above the $\lambda$-point. On contrary, experiment $\underline{\underline{3}}$ gives only very weak change in the temperature dependence of the surface tension at the $\lambda$-point, suggesting that the ripplon contribution is not the main one. In liquid ${ }^{3} \mathrm{He}$ the viscosity is much higher than in ${ }^{4} \mathrm{He}$, and only the very long-wavelength ripplons with energy $\hbar \omega_{k} \ll T$ survive at $T<1 K$. These long-wavelength ripplons give negligible contribution to the free energy because of the small number of quantum states, which is proportional to the phase volume. Hence, the theory predicts a very weak dependence $\alpha(T)$ for ${ }^{3} \mathrm{He}$ in the whole temperature interval, which strongly contradicts the experimental observations $\stackrel{4}{*}$. This discrepancy between the theory and experiment on the liquid helium surface tension 
remained a puzzle for several decades, until the new type of surface excitations has been proposed ${ }^{7}$ This new type of excitations, called the surface level atoms (SLA) or surfons, allowed to explain the temperature dependence of the surface tension and to reach the very high agreement between theory and experiment ${ }^{7}$ The surfons resemble the states of ${ }^{3} \mathrm{He}$ atoms on the surface of liquid ${ }^{4} \mathrm{He}$ in the ${ }^{3} \mathrm{He}-{ }^{4} \mathrm{He}$ mixtures 8 and the states of ${ }^{3} \mathrm{He}$ or ${ }^{4} \mathrm{He}$ atoms on the surface of liquid hydrogen ${ }^{9}$ The surface states in the ${ }^{3} \mathrm{He}-{ }^{4} \mathrm{He}$ mixtures, called the Andreev states, were also introduced to explain the temperature dependence of the surface tension of these mixtures. The main difference between the Andreev states and the surfons is that the latter exist even in the pure He isotopes.

A thorough microscopic description of this new type of excitations is a rather complicated manyparticle problem. In Ref $\underline{7}$ the surfons were considered phenomenologically as the quantum states of helium atoms localized at the liquid helium surface. The surfons may propagate in the surface plane and have the quadratic dispersion

$$
\varepsilon(k)=E_{s}+k^{2} / 2 M^{*}
$$

where $k$ is the $2 \mathrm{D}$ momentum of surfons along the surface. Both the surfon energy $E_{s}$ and their effective mass $M^{*}$ depend on the He isotope ${ }^{3} \mathrm{He}$ or ${ }^{4} \mathrm{He}$. The surfon energies $E_{s}$ are intermediate between the energy of a He atom in vacuum $E_{v a c}^{H e}$ and the chemical potential $\mu$ of this atom inside the liquid. If one takes the energies of He atoms in vacuum as the reference point, $E_{v a c}^{H e}=0$, the chemical potentials of liquid ${ }^{4} \mathrm{He}$ and ${ }^{3} \mathrm{He}$ at $T \rightarrow 0$ are

$$
\mu_{0}^{H e 4}=-7.17 K, \text { and } \mu_{0}^{H e 3}=-2.5 K .
$$

The creation of a surfon is a thermal activation process with the activation energy

$$
\Delta(T)=E_{s}-\mu(T) .
$$

Therefore, at low enough temperature $T \ll \Delta$ the concentration of surfons is exponentially small. Fitting the experimental data on the temperature dependence of the surface tension, below we obtain the values of this activation energy for ${ }^{4} \mathrm{He}$ and ${ }^{3} \mathrm{He}$ :

$$
\Delta^{H e 4} \approx 2.67 K, \Delta^{H e 3} \approx 0.25 K .
$$

The corresponding energies of surfons are

$$
E_{s}^{H e 4} \approx-4.5 K \text { and } E_{s}^{H e 3} \approx-2.25 K,
$$

The effective masses, obtained from this fitting, are

$$
M_{4}^{*} \approx 2.65 M_{4}^{0} \text {, and } M_{3}^{*} \approx 2.25 M_{3}^{0},
$$

where $M_{4}^{0}$ and $M_{3}^{0}$ are the masses of free ${ }^{4} \mathrm{He}$ and ${ }^{3} \mathrm{He}$ atoms.

The role of surfons may be crucial for various properties of electrons on the liquid helium surface (see Refs $10,11,12$ for reviews of this area). Electron scattering on surfons reduces the mobility of the electrons. 13 This scattering also affects the electron transitions between the bound states on the surface, leading to the shift and the broadening of the transition line 11,14 This is particularly important for the physical realization of quantum bits and quantum computing with the use of electrons on the liquid helium surface. 15

In the present paper we microscopically substantiate the existence of surfons and study their properties. We estimate the energy (6) ant the effective mass (7) of surfons from the microscopic considerations. We show that taking into account the temperature dependence of the chemical potential of liquid helium allows to reach the agreement between theory and experiment on the dependence $\Delta \alpha(T)$ up to the accuracy of the existing experiments. We propose the theoretical curve of this dependence in the entire temperature interval from zero to the boiling temperature $T_{c}$ of liquid helium. This theoretical prediction explains the existing puzzles in the temperature dependence of the surface tension of liquid helium and other liquids, and stimulates further experiments.

\section{ESTIMATION OF THE SURFON ACTIVATION ENERGY AND EFFECTIVE MASS}

The goal of this section is to prove the existence of the surfons from the quantum-mechanical calculation and to give a rough estimate of its activation energy and effective mass. In the next section we find these values phenomenologically with higher accuracy from the comparison with experiment on the 
temperature dependence of the surface tension $\Delta \alpha(T)$. In this section we use the simplified interaction between He atoms, which includes only the Hartree term of the van der Waals forces and neglects the exchange interaction. Then we solve the one-particle Schrödinger equation for the surface atom in the potential, formed by other atoms, taking into account possible formation of a dimple (or polaron) under the atom on the surface.

\section{A. The surfon activation energy levels on the flat surface}

The interaction potential between two helium atoms can be described by the Lennard-Jones (LJ) potential 16

$$
V_{L J}(r)=4 \epsilon_{0}\left[\left(\sigma_{0} / r\right)^{12}-\left(\sigma_{0} / r\right)^{6}\right],
$$

where the generally accepted coefficients

$$
\epsilon_{0}=10.22 K \text { and } \sigma_{0}=2.556 \AA
$$

are the same for both He isotopes and obtained by fitting the He atom scattering experiments. This potential includes the van-der-Waals attraction between atoms at long distance and the hard-core repulsion at short distance. More complicated He-He interatomic potentials have been proposed by various authors,$\underline{17}$ but for our estimates the accuracy of the potential (8) is safficient. The potential energy of an atom above the liquid can be calculated by the summation of the interatomic potential over all atoms in the liquid. This approximation neglects the many-particle effects and the back influence of an atom above the surface on the bulk liquid, studied later. The integration of the Lennard-Jones potential (8) over the uniform liquid in the half-space $z<0$ gives the following potential energy of an atom above the surface of liquid:

$$
V(z)=\frac{4 \pi \epsilon_{0} \sigma_{0}^{3} n_{b}}{3}\left[\frac{1}{15}\left(\frac{\sigma_{0}}{z}\right)^{9}-\frac{1}{2}\left(\frac{\sigma_{0}}{z}\right)^{3}\right] .
$$

Here $z$ is the distance of the atom from the surface, $n_{b}$ is the number atom density in the bulk liquid. This bulk density in liquid ${ }^{4} \mathrm{He}$ at $T \rightarrow 0$ is $n_{\mathrm{He} 4}=0.02186 \AA^{-3}$, and in ${ }^{3} \mathrm{He} n_{\mathrm{He}}=0.0164 \AA^{-3}$. The potential (9) is attractive at long distance $z>z_{\min }=\sigma_{0}(2 / 5)^{1 / 6} \approx 2.194 \AA$ and repulsive at $z<z_{\min }$. The substitution of the numbers to Eq. (9) gives the following potentials of He atoms above ${ }^{4} \mathrm{He}$ and ${ }^{3}$ He surfaces:

$$
\begin{aligned}
V^{H e 4}(z) & =\left(\frac{4852}{z^{9}}-\frac{130.5}{z^{3}}\right)[K], \\
V^{H e 3}(z) & =\left(\frac{3640}{z^{9}}-\frac{97.9}{z^{3}}\right)[K] .
\end{aligned}
$$

The numerical solution of the one-dimensional Schrödinger equation for a He atom in these potentials proves the existence of discrete energy levels for both He isotopes, and gives the following estimates for the energy of this bound state in the zeroth approximation:

$$
E_{s 0}^{H e 4} \approx-1.24 K \text {, and } E_{s 0}^{H e 3} \approx-0.342 K .
$$

These calculated values are higher than the values (6) obtained from the surface tension data fit, which is not surprising. First, the above model takes the liquid surface to be rigid and flat, while in fact this surface is very soft and subjected to deformation, which reduces the bound state energy (12). Second, the distribution of He atoms in the liquid is not uniform and is affected by an atom on the surface level. One expects the increase of liquid density around the surfons. Third, the identity of the atom on the surface level with the atoms in the bulk liquid leads to the exchange energy correction and other many-body effects $\frac{18}{18}$

The similar problem appears in the calculation of the energy of Andreev levels,,$\frac{8}{\text { i.e. }}$ of the bound states of ${ }^{3} \mathrm{He}$ atoms on the surface of liquid ${ }^{4} \mathrm{He}$. The solution of $1 \mathrm{D}$ Schrödinger equation with the potential (9) gives the energy of bound state $E_{A} \approx-0.8 K$, while the value of Andreev energy levels, as obtained from the surface tension experiments, is about $E_{A}^{\exp } \approx-5 K$. Various approaches have been developed to calculate the Andreev energy levels more accurately,$\underline{2}$ but the substantial disagreement between theoretical and experimental results still exists. 
B. Formation of a dimple and its influence on the surfon activation energy and effective mass.

In this section we study the back influence of the surfons on the bulk liquid. In the first approximation, one must consider the formation of a dimple under the surfon, similar to the dimple under an electron $10,11,12$ or negative ion $\frac{19}{2}$ on the liquid helium surface. In the next approximation one also needs to account for the increase of liquid density in the vicinity of surfon. This adjustment of the liquid to the appearance of the surfon is similar to the polarons around electrons in solids ${ }^{20}$ The surface deformation is a polaron of ripplons, while the increase of the liquid density under the surfon is a polaron of bulk phonons. This composite polaron propagates with the surfon along the liquid surface, reducing the surfon activation energy and increasing its effective mass. Since the relative variation of the liquid density around the surfon is small, the correction to the surfon activation energy from the density polaron is also small. On contrary, the surface deformation around the surfon may considerably change the van der Waals potential at the surface, and this effect must be taken into account.

For the estimate of the correction to the surfon energy and its effective mass due to the dimple on the liquid surface, we apply the methods similar to those in the theory of electrons $10,11,12$ and negative ions $\frac{19}{9}$ on the liquid helium surface, replacing the electrostatic attraction by the van-der Waals forces. The formation of a dimple on the surface under the surfon gains the van-der Waals energy $E_{W}$ but costs the surface tension energy $E_{\text {surf }}$ and the quantum kinetic energy $E_{k i n}$ of the surfon due to the additional confinement of its wave function near the dimple. All these contributions depend on the shape of the dimple and determine this shape. Their self-consistent calculation requires the solution of the axially-symmetric 3D Schrödinger equation for He atom above the dimple. We do not perform the complicated calculation of the dimple profile, because the model of a classical static surface deformation itself is rather rough. Instead, we estimate the energy gain due to the formation of the dimple taking its shape $\Delta z(x, y)=\xi\left(\rho \equiv \sqrt{x^{2}+y^{2}}\right)$ to be a spherical cap of radius $R$ and depth $h$ :

$$
\xi(\rho)=\left\{\begin{array}{cl}
R-h-\sqrt{R^{2}-\rho^{2}}, & \rho<\sqrt{R^{2}-(R-h)^{2}} \\
0, & \rho \geq \sqrt{R^{2}-(R-h)^{2}}
\end{array}\right.
$$

The loss in the surface tension energy $E_{\text {surf }}$ of the shallow dimple of depth $h \ll R$ is approximately proportional to $h^{2}$ and depends weakly on the shape of the dimple. Thus, for the spherical dimple (13)

$$
E_{\text {surf }}=\pi h^{2} \sigma
$$

while for the much smoother Gaussian shape $\xi(\rho)=-h \exp \left(-\rho^{2} / 2 R^{2}\right)$ with the same depth and curvature in the center, the surface energy loss reduces by one half: $E_{\text {surf }}^{\text {Gaus }}=\pi h^{2} \sigma / 2$. The van der Waals attraction is a very short-range one. Therefore, the optimal dimple does not extend far from the surfon. The optimal dimple radius is determined from the competition between the van der Waals attraction $E_{W}$ and the kinetic energy $E_{k i n}$. This competition also happens in the bulk liquid, and leads to the mean inter-particle distance $d=n_{b}^{-1 / 3}=3.58 \AA$ for ${ }^{4} \mathrm{He}$ and $d=3.94 \AA$ for ${ }^{3}$ He. Below, we take the dimple curvature radius $R=d$ in our estimate of the SLA energy correction. The depth of the dimple is determined from the competition between the surface tension energy $E_{\text {surf }}$ and the van der Waals energy $E_{W}$.

The van der Waals energy gain from the dimple (13) can be estimated by the integration of the LJ potential (8) over the region $0<z<h$

$$
E_{W}=\int d^{3} \mathbf{r} n_{b}(\mathbf{r}) \int \Psi_{s}^{2}\left(\mathbf{r}^{\prime}\right) d^{3} \mathbf{r}^{\prime} V_{L J}\left(\mathbf{r}-\mathbf{r}^{\prime}\right)
$$

The kinetic energy $E_{k i n}<\left|E_{W}\right|$ already implicitly enters Eq. (14) through the surfon wave function $\Psi_{s}(\mathbf{r})$ and the minimal dimple radius $d$. Its additional contribution reduces the energy gain (14) by the factor $\sim 1$. However, we neglect this correction because we did not account for the similar factor in the surface tension energy loss. We also reduce the van der Waals energy gain by similar factor by taking the wave function in the integral (14) in the form of $\delta$-function: $\Psi_{s}^{2}\left(\mathbf{r}^{\prime}\right)=\delta^{3}\left(\mathbf{r}^{\prime}-\mathbf{r}_{0}\right)$, where $\mathbf{r}_{0}$ is the position of the center of surfon. Thus, we have

$$
E_{W}+E_{k i n} \approx n_{b} \int_{0}^{h} d z \int_{\rho_{0}(z)}^{\infty} \pi d \rho^{2} V_{L J}(r)
$$


where $\rho_{0}^{2}(z)=R^{2}-(R-h+z)^{2} \approx 2 R(h-z)$ and $r^{2}=(R-h+z)^{2}+\rho^{2}$. Integration gives

$$
\frac{E_{W}+E_{k i n}}{4 \pi \epsilon_{0} \sigma_{0}^{2} n_{b}} \approx h\left[\frac{1}{5}\left(\frac{\sigma_{0}^{2}}{R^{2}}\right)^{5}-\frac{1}{2}\left(\frac{\sigma_{0}^{2}}{R^{2}}\right)^{2}\right] .
$$

For ${ }^{4} \mathrm{He}$ this gives $E_{W} \approx-2.3 h$ and for ${ }^{3} \mathrm{He}$ this gives $E_{W} \approx-1.2 h$, where the dimple depth $h$ is in $\AA$ and $E_{W}$ is in $K$. Minimization of

$$
\Delta E_{s} \approx E_{W}+E_{k i n}+E_{\text {surf }}
$$

gives for ${ }^{4} \mathrm{He}$ the optimal dimple depth and energy gain

$$
h_{4} \approx 1 \AA \text { and } \Delta E_{s}^{H e 4} \approx-1.2 K
$$

and for ${ }^{3} \mathrm{He}$

$$
h_{3} \approx 1.2 \AA \text { and } \Delta E_{s}^{H e 3} \approx-0.73 K \text {. }
$$

The surfon effective mass increases due to the dimple. This increase can be roughly estimated assuming that during the surfon motion on the interatomic distance $d$ at least all adjacent atoms must also move by the distance of the order of the dimple depth. Then, the surfon effective mass

$$
m^{*} \approx m_{H e}\left(1+n_{a d j} h / d\right) .
$$

The number of adjacent atoms $n_{a d j} \sim 6$, and we get

$$
M_{4}^{*} \approx 3 M_{4}^{0}, \text { and } M_{3}^{*} \approx 2 M_{3}^{0} .
$$

The above rough calculation is aimed to show that the surface deformation under the surfon is essential both for the surfon activation energy. For more accurate study of the surfon parameters, in the next section we compare with experiment the thermodynamical properties of liquid surface, calculated using the above model of surfons.

\section{TEMPERATURE DEPENDENCE OF LIQUID HELIUM SURFACE TENSION.} is $\frac{5}{5}$

The contribution $\Delta \alpha_{S}(T)$ of the $2 \mathrm{D}$ surfon gas to the temperature dependence of the surface tension

$$
\Delta \alpha_{S}(T)= \pm g T \int \ln \left[1 \pm \exp \left(\frac{\mu-\varepsilon(k)}{T}\right)\right] \frac{d^{2} k}{(2 \pi \hbar)^{2}}
$$

This is just a contribution of the $2 \mathrm{D}$ gas of noninteracting particles with dispersion $\varepsilon(k)$ to the free energy of unit surface area.$^{\frac{5}{5}}$ Here $\mu=\mu(T)$ is the temperature-dependent chemical potential of liquid helium, $\varepsilon(k)$ is the dispersion of surfons, $g$ is the spin degeneracy ( $g=1$ for ${ }^{4} \mathrm{He}$ and $g=2$ for ${ }^{3} \mathrm{He}$ ), and the sign " $\pm "$ in (19) is " -" for bosons and " +" for fermions. The surfons above ${ }^{4} \mathrm{He}$ are the bosons with dispersion (2). After introducing the new variable $z \equiv \exp \left[-k^{2} / 2 M^{*} T\right]$, their contribution to the surface tension becomes

$$
\Delta \alpha_{4}(T)=\frac{T^{2} M_{4}^{*}}{2 \pi \hbar^{2}} \int_{0}^{1} \frac{\ln \left[1-z \exp \left(-\Delta_{4} / T\right)\right]}{z} d z
$$

where

$$
\Delta_{4} \equiv \Delta_{4}(T)=E_{s}^{H e 4}-\mu_{4}(T) .
$$

Above ${ }^{3} \mathrm{He}$ the surfons are fermions, and from (19) we obtain after the integration by parts

$$
\Delta \alpha_{3}(T)=-\frac{M_{3}^{*} T^{2}}{\pi \hbar^{2}} \int_{0}^{1} \frac{\ln \left[1+z \exp \left(-\Delta_{3} / T\right)\right]}{z} d z,
$$


where

$$
\Delta_{3} \equiv \Delta_{3}(T)=E_{s}^{H e 3}-\mu_{3}(T) .
$$

From (20), (22) we see that the contribution of surfons to $\alpha_{3,4}(T)$ depends exponentially on temperature at low $T: \Delta \alpha \propto T^{2} \exp (-\Delta / T)$.

To calculate the integrals in (20), (22) one need to know the temperature dependence of the chemical potential $\mu(T)$, which enters Eqs. (20) and (22) via the surfon energy gap $\Delta$. In Ref $\stackrel{7}{=}$ this dependence has been considered only for ${ }^{3} \mathrm{He}$ and only qualitatively using the approximate formula (20) of Ref $\underline{\underline{7}}$. For quantitative analysis in the wider temperature range we take the dependence $\mu(T)$ from the experiment. We take the exact formula for the chemical potential

$$
\mu(T)=\frac{F_{V}(T)+P(T)}{n_{L}(T)} .
$$

Here the pressure $P(T)$ is equal to the saturated vapor pressure, and the temperature-dependent particle density $n_{L}$ in liquid He is determined from the mass density. The free energy per unit volume is

$$
F_{V}(T)=\int_{0}^{T} C_{V}(T) d T-T S_{V}(T)
$$

where the heat capacity $C_{V}$ and the entropy $S_{V}$ at constant volume are also taken from experiment. For ${ }^{4} \mathrm{He}$ the data on the temperature dependence of the quantities $C_{V}, S_{V}, P, n_{L}$ entering Eqs. (24), (25) are taken from Ref. 21 . For ${ }^{3} \mathrm{He}$ the data on $C_{V}(T)$ and $S_{V}(T)$ are taken from Ref $\stackrel{22}{,}$, and the data on saturated pressure are taken from Ref $\underline{23}$. The data on the temperature dependence of the chemical potential and of the surfon activation energy are plotted in Figs. 1] and 5. Note that for both helium isotopes the experimentally measured temperature dependence of the chemical potential is in a high agreement with the predictions of the theory of quantum nondegenerate liquids, developed in Refs $\stackrel{24,25}{.}$.

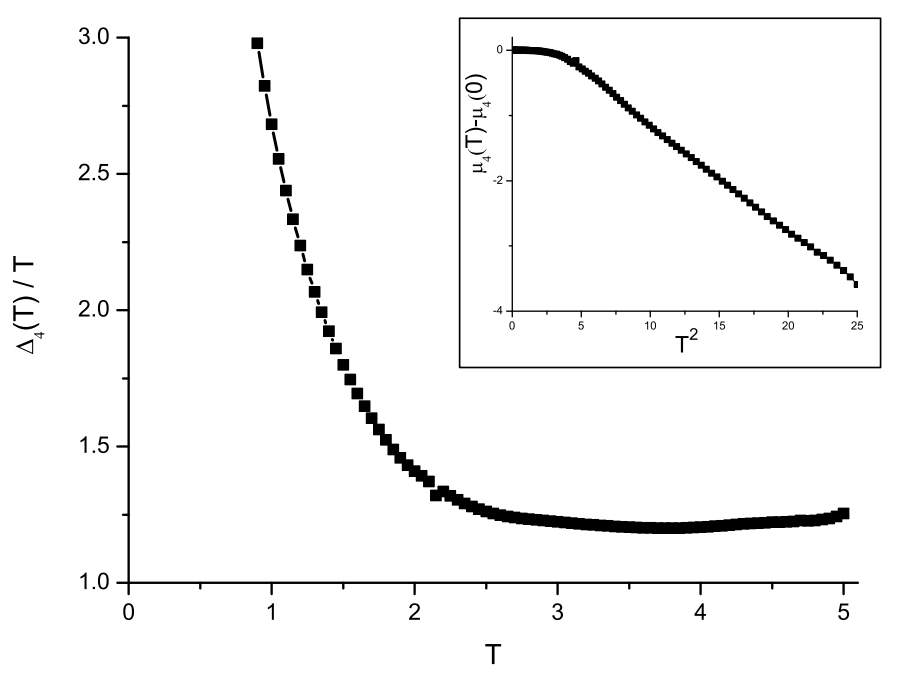

FIG. 1: The temperature dependence of surfon gap $\Delta_{4}(T)$ for liquid ${ }^{4}$ He divided by temperature, as it enters Eq. (20). This dependence is extracted from the experimental temperature dependence of chemical potential $\mu_{4}(T)-\mu_{4}(0)$ of liquid ${ }^{4} \mathrm{He}$, shown in the insert figure and obtained from the data in Ref ${ }^{21}$. The graph shows that $\Delta(T) / T$ is almost independent of temperature in the wide range $T_{\lambda}<T \lesssim 4 K$. Such a dependence of $\Delta(T)$, being substituted in to Eq. (20), explains the quadratic temperature dependence of the surface tension at $T>T_{\lambda}$.

In the superfluid ${ }^{4} \mathrm{He}$ at $T<T_{\lambda}=2.17 \mathrm{~K}$ the chemical potential $\mu(T)$ depends weakly on temperature. At $T>T_{\lambda}$ this dependence is quadratic (see insert of Fig. 11) in accordance with the theoretical prediction $\underline{24}$

$$
\mu_{4}(T)=\mu_{4}(0)+\varepsilon_{4}^{0}-T^{2} / T_{4}^{0}
$$




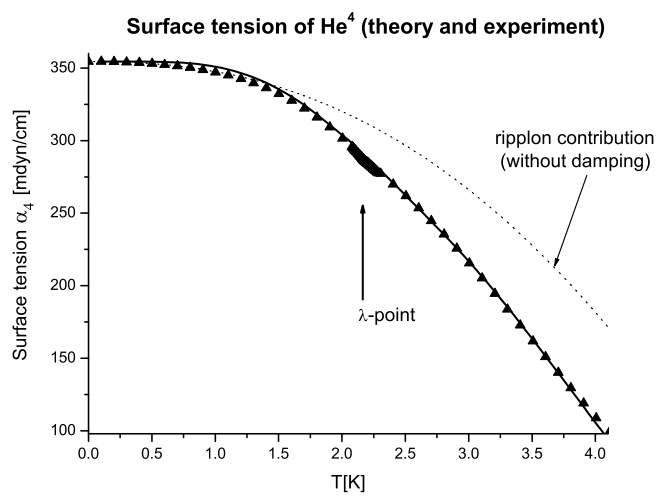

FIG. 2: The calculated surfon contribution to the temperature dependence of the surface tension of ${ }^{4} \mathrm{He}$ (solid line) compared with the experimental data from Ref. ${ }^{3}$ (black triangulares). At $T>T_{\lambda}$ the agreement is so high that in the linear scale the deviation cannot be detected by eye. In this temperature region the ripplons are damped by viscosity, and their contribution to the surface tension $\Delta \alpha_{4}(T)$ is negligible. At low temperature, the concentration of surfons is exponentially small due to the activation energy $\Delta^{H e 4} \approx 2.7 K$, and the main contribution to $\Delta \alpha_{4}(T)$ comes from the gapless ripplons. The dotted line represents Eq. (1), which gives the ripplon contribution without viscosity and is valid only deep inside the superfluid phase. This line agrees with the experimental data only at $T<1.5 \mathrm{~K}$.

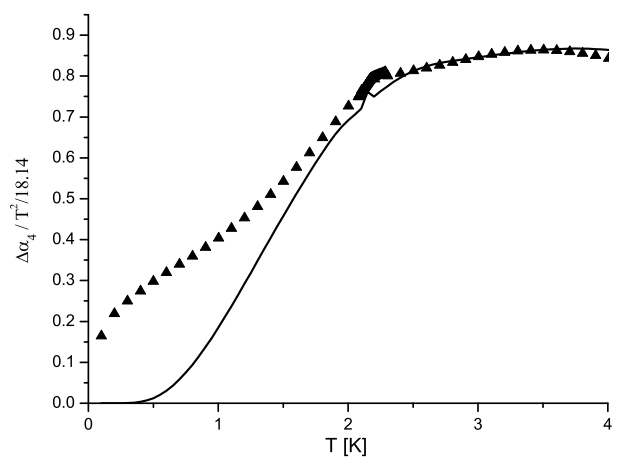

FIG. 3: The temperature dependence of the surface tension of ${ }^{4} \mathrm{He}$ divided by $T^{2}$. This graph shows the comparison between our theory and experiment in the coordinates where the difference can be visually observed. The low-temperature deviation at $T<T_{\lambda}$ is due to the ripplon contribution, while the deviation at $T>4 K$ is due to the proximity to the boiling point. The ripplon contribution at $T<T_{\lambda}$, extracted from this graph, is shown in Fig. 4

where $\varepsilon_{4}^{0}=0.55 \mathrm{~K}$ and $T_{4}^{0}=5.59 \mathrm{~K}$. The function $\Delta_{4}(T) / T$, which determines the temperature dependence of the surface tension $\Delta \alpha_{4}(T)$ in (20) and is given by Eq. (21), depends weakly on temperature in the interval $T_{\lambda}<T<4.5 K$ (see Fig. 1). The minimum of the function $\Delta(T) / T$ occurs at $T \approx 3.5 K$, but in the entire range $T_{\lambda}<T<4.5 K$ one can take $\Delta(T) / T=$ const with the accuracy $\sim 4 \%$. Then the integrand in Eq. (20) does not depend on temperature, which results in the quadratic temperature dependence of the surface tension of liquid ${ }^{4} \mathrm{He}$ with the same accuracy $\sim 4 \%$ (see Figs. 213). This explains the long-standing puzzle of the experimentally observed quadratic temperature dependence of liquid ${ }^{4} \mathrm{He}$ at $T>T_{\lambda}$. The values $\Delta_{4}=2.67 \mathrm{~K}$ and $M_{4}^{*}=2.65 M_{4}^{0}$ give the best fit to the experimental points. The ripplon contribution to the temperature dependence of the surface tension $\Delta \alpha_{4}(T)$ is small and has maximum at $T \approx 1.1 K$ (see Fig. (4), where it makes only $\sim 2 \%$ of $\alpha_{4}(T)$ (see Figs. [2]3). In Ref. $\underline{7}$ it was shown that at $T>T_{\lambda}$ the thermal ripplons with energy $\hbar \omega_{k} \sim T$, which give the main contribution to $\Delta \alpha_{4}(T)$, are strongly damped by viscosity. In ${ }^{3} \mathrm{He}$ the short-wave-length thermal ripplons are strongly damped in the whole temperature range. Therefore, the ripplon contribution to $\Delta \alpha_{4}(T)$ is small. The long-wave-length ripplons with energy $\hbar \omega_{k} \ll T$ are not damped by viscosity, but their contribution to $\Delta \alpha_{4}(T)$ is small because of the small phase volume.

The temperature dependence of the chemical potential $\mu_{3}(T)$ in ${ }^{3} \mathrm{He}$, obtained using Eqs. (24), (25) 


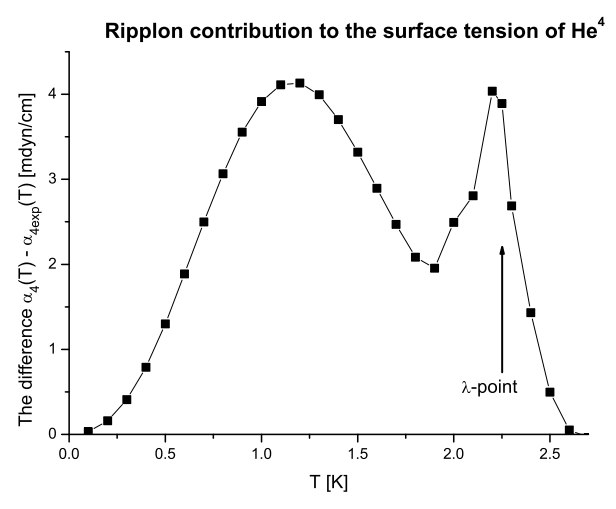

FIG. 4: The difference between the surfon contribution $\alpha_{4}(T)$ to the surface tension of ${ }^{4} \mathrm{He}$, given by Eq. (20), and the experimental values $\alpha_{4 \exp }(T)$. The maximum at $T \approx 1.1 K$ is due to the ripplon contribution. The second maximum at $T \approx 2.17 K$ corresponds to phase transition at the $\lambda$-point of ${ }^{4} \mathrm{He}$.

from the experimental data in Ref. $\underline{22}$, is shown in Fig. [5. At $T>0.3 K$, where ${ }^{3} \mathrm{He}$ is nondegenerate, this dependence with very high accuracy coincides with the theoretical prediction ${ }^{24}$

$$
\mu_{3}(T)=\mu_{3}(0)-T \ln 2+\varepsilon_{3}^{0}-T^{2} / T_{3}^{0}
$$

where

$$
\varepsilon_{3}^{0}=0.28 \mathrm{~K} \text { and } T_{3}^{0}=4.83 \mathrm{~K} \text {. }
$$

At $T<0.3 K$, when ${ }^{3} \mathrm{He}$ is a degenerate Fermi liquid,

$$
\mu_{3}(T) \approx \mu_{3}(0)-T^{2} / 2 T_{F},
$$

where $T_{F}=0.36 \mathrm{~K}$. Note, that the quadratic temperature dependence of the chemical potential [see Eqs. (26),(27)] is the characteristic feature of all quantum nondegenerate liquids. ${ }^{24,25}$ Such temperature dependence $\mu(T)$ also takes place in liquid hydrogen $\stackrel{\underline{14}}{=}$ An alternative theory of this dependence is given in $\operatorname{Ref}^{26}$.

Similarly to the case of ${ }^{4} \mathrm{He}$, in ${ }^{3} \mathrm{He} \Delta_{3}(T) / T$ is almost independent of temperature with accuracy $\sim 5 \%$ in the wide temperature range $0.3 K<T<2.5 K$ (see insert in Fig. 5). According to Eq. (22), this occasional feature of the function $\Delta_{3}(T) / T$ results in the nearly quadratic dependence $\Delta \alpha_{3}(T)$ in the same temperature interval (see Fig. 6). From comparison with the experiment on $\Delta \alpha_{3}(T)$ in Ref. $\underline{\underline{4}}$ we obtain the values $\Delta_{3}(0)=0.25 K$ and $M_{3}^{*} \approx 2.25 M_{3}^{0}$.

One sees, that the account for the temperature dependence of the chemical potential substantially improves the agreement between the proposed theory and the experimental data on $\Delta \alpha(T)$ for both helium isotopes and extends the temperature interval of this agreement to the whole interval of the liquid phase (see Figs. 22 and 6). According to the above calculations and to the experimental data, in a wide temperature range the deviation of the surface tension coefficient $\Delta \alpha_{3}(T) \propto T^{2}$ and $\Delta \alpha_{4}(T) \propto T^{2}$. This shows that the particle statistics of surfons is not very important for the surface phenomena on the liquid helium. The extracted parameters of the surfon activation energy and their effective mass are in a reasonable agreement with the theoretical predictions (15), (16), (18) of our rough model and can be used for more elaborated theoretical study of the structure of this new type of surface excitations.

\section{DISCUSSION AND SUMMARY}

In Sec. II we proposed a simple theoretical model of the new type of surface excitations (called surfons), and perform the quantum-mechanical calculation of the surfon activation energy $E_{s}$ and their effective mass $M^{*}$ basing on this model. This calculation substantiates the existence of surfons, because it shows that He atoms have at least one discrete energy level at the liquid surface. He atoms are attracted to the surface by the van der Waals force and become localized in the direction, perpendicular to the surface, propagating only in the surface plane. As the result, they form a 2D gas on the liquid surface. The temperature-dependent concentration of the surfons is determined by their activation 


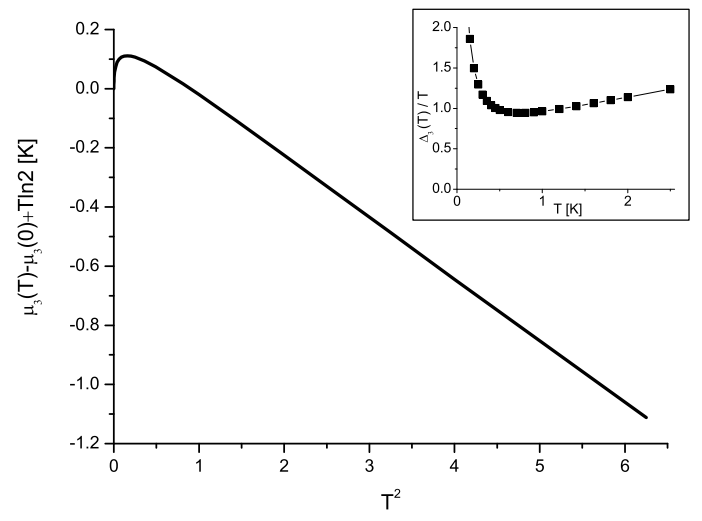

FIG. 5: The experimental temperature dependence of the chemical potential $\mu_{3}(T)-\mu_{3}(0)+T \ln 2$, obtained from the data in Ref. ${ }^{22}$ and plotted as function of $T^{2}$ to show that Eq. (27) is fulfilled with high accuracy. The insert figure shows the temperature dependence of the surfon gap $\Delta_{3}(T)$ divided by temperature. One sees that $\Delta_{3}(T) / T$ is almost independent of $T$ in the wide temperature interval $0.3 K<T \lesssim 2.5 K$. This dependence, substituted to Eq. (22), explain the quadratic temperature dependence of the surface tension $\Delta \alpha_{3}(T)$ at $T>0.3 K$.

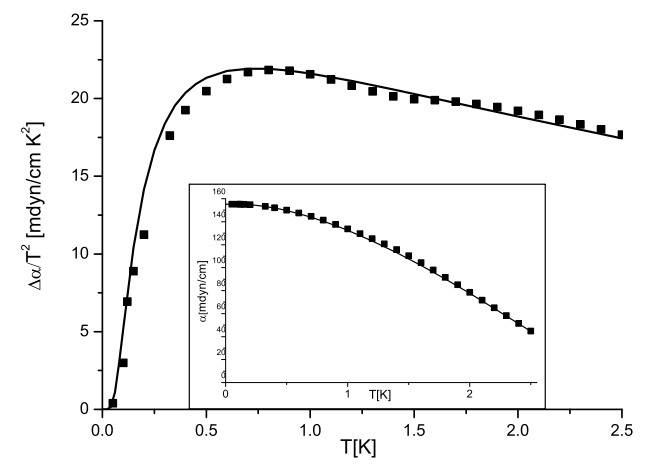

FIG. 6: The temperature dependence of the surface tension of ${ }^{3} \mathrm{He}$, calculated using Eqs. (22), (23) (solid line) compared to the experimental data from Ref. ${ }^{4}$ (black squares). The agreement is so high that in the linear scale (insert figure) the deviation cannot be detected by eye. Therefore, on the main figure we plot $\Delta \alpha_{3} / T^{2}$. The agreement between theory and experiment considerably improves after taking the experimental dependence of $\mu(T)$ into account.

energy $\Delta=E_{s}-\mu$ [see Eq. (44)]. If $E_{s}<\mu$, the liquid is unstable. If $E_{s}>0$, the concentration of surfons in negligibly small compared to the concentration of helium vapor at any temperature. For both He isotopes, the calculated values $E_{s}$ lie in the interval $\mu<E_{s}<0$. The formation of a dimple under the surfon reduces the surfon activation energy by the value $\Delta E_{s} \sim 1 K$ [see Eqs. (15), (16)]. The dimple also increases several times the effective mass of the surfons in their in-plane motion [see Eqs. (17), (18)]. This increase is not surprising, because during the in-plane motion the surfon also drags the dimple, which includes the motion of several atoms in the liquid.

The experimental observation and investigation of surfons is possible because the surfons make the main contribution to the temperature dependence of the surface tension. In Sec. III we calculate this contribution, taking into account the temperature dependence of the chemical potential and liquid density, which we derive from the experimental data. This considerably improves the agreement between our theory and experiment of the temperature dependence of the surface tension as compared to the previous letter ${ }^{7}$, making the deviations to be as small as $\lesssim 1 \%$ in the whole temperature range [for ${ }^{4} \mathrm{He}$ this agreement is achieved after the inclusion of ripplon contribution, given by Eq. (11) at temperature below the $\lambda$-point]. The two extracted fitting parameters, the surfon activation energy $\Delta$ and the effective mass $M^{*}$, are in a reasonable agreement with our calculations in Sec. II. Their values are given 
by Eqs. (5)-(17). At very low temperature the surfon contribution to the temperature dependence of the surface tension is small, because the concentration of surfons is exponentially small being determined by the activation energy $\Delta$. At high temperature the surfon contribution $\propto T^{2}$ for both helium isotopes.

For even higher agreement between theory and experiment on the temperature dependence of the surface tension we also considered the interaction between surfons. At low temperature this interaction is not important, because the concentration of surfons is low. At higher temperature the interaction between surfons only renormalizes the surfon effective mass. The details of these results will be published elsewhere.

\section{ACKNOWLEDGEMENT}

A.G. thanks Prof. A.F. Krutov for useful discussions. The work was supported by the Foundation "Dynasty" and by the visitor program of Max Planck Institute for the Physics of Complex Systems.

* Visiting address: Max Planck Institute for the Physics of Complex Systems, Dresden D-01187 Germany

† Electronic address: grigorev@itp.ac.ru

1 J.S.Rowlinson and B. Widom, Molecular Theory of cappilarity, Dover Publications, Mineola NY, 2002.

2 D.O. Edwards and W.F. Saam, Chapter 4 in The free surface of liquid helium, Ed. by D.F. Brewer, Progress in Low Temperature Physics (series), North-Holland Publishing Company, 1978

${ }^{3}$ M. Iino, M. Suzuki, and A.J. Ikushima, J. Low Temp. Phys. 61, 155 (1985).

${ }^{4}$ M. Iino, M. Suzuki, A.J. Ikushima and Y. Okuda, J. Low Temp. Phys. 59, 291 (1985); M. Suzuki, Y. Okuda, A.J. Ikushima and M.Iino, Europhys. Lett. 5, 333 (1988); K. Matsumoto, Y. Okuda, M. Suzuki and S. Misawa, J. Low Temp. Phys. 125, 59 (2001).

${ }^{5}$ L. D. Landau and E. M. Lifshitz, Course of Theoretical Physics, Vol. 5: Statistical Physics, 3rd ed. (Nauka, Moscow, 1976; Pergamon Press, Oxford, 1980).

${ }^{6}$ K. R. Atkins, Can. J. Phys. 31, 1165 (1953).

7 A.M. Dyugaev, P.D. Grigoriev, JETP Lett. 78, 466 (2003) [Pisma v ZhETF 78, 935 (2003)].

8 A.F. Andreev, JETP 23, 939 (1966) [Zh. Exp. Teor. Fiz. 50, 1415 (1966)].

${ }^{9}$ C.G. Paine and G.M. Seidel, Phys. Rev. B 46, 1043 (1992).

10 V.B. Shikin and Yu.P. Monarkha, Two-Dimensional Charged Systems in Helium (in Russian), Nauka, Moscow (1989).

11 V.S. Edel'man, Sov. Phys. - Uspehi 130, 676 (1980).

12 Y. Monarkha, K. Kono, Two-Dimensional Coulomb Liquids and Solids, Springer Verlag, 2004.

13 P.D. Grigoriev, A.M. Dyugaev, E.V. Lebedeva, JETP 106(2), 316 (2008).

14 P. D. Grigor'ev, A. M. Dyugaev and E. V. Lebedeva, JETP Letters 87, 106 (2008) [Pisma v ZhETF 87, 114 (2008)].

15 P.M. Platzman, M.I. Dykman, Science 284, 1967 (1999); M. I. Dykman, P. M. Platzman, and P. Seddighrad, Phys. Rev. B 67, 155402 (2003).

16 J. E. Lennard-Jones, Proc. Roy. Soc. A 106, 463 (1924).

17 R. Hellmann, E. Bich and E. Vogel, Molecular Physics, 105, 3013 (2007); R. A. Aziza and M. J. Slaman, J. Chem. Phys. 94 (12), 8047 (1991). D.E. Nitz, D.Sieglaff, M. Lagus et al., Phys. Rev. A 47, 3861 (1993).

18 This exchange energy correction is expected to be small and less important for the value of the surface energy level than the local adjustment of the atoms in the liquid to the surfon. The small value of the exchange energy comes from the very small overlap of the wave functions of He atoms due to their hard-core repulsion.

19 P. D. Grigor'ev and A. M. Dyugaev, JETP 88, 325 (1999) [ZhETF 115, 593 (1999)].

20 G. Mahan, Many-Particle Physics, 2nd ed., Plenum Press, New York, 1990.

21 Russell J. Donnelly and Carlo F. Barenghi, The Observed Properties of Liquid Helium at the Saturated Vapor Pressure, Journal of Physical and Chemical Reference Data 27, 1217 (1998).

22 D.S. Greywall, Phys. Rev. B 27, 2747 (1983).

23 Stephen G. Sydoriak and Thomas R. Roberts, Phys. Rev. 106, 175 - 182 (1957); Jost Engert, Bernd Fellmuth and Karl Jousten, Metrologia 44, 40 (2007).

24 A.M. Dyugaev, J. Low Temp. Phys. 78, 79 (1990).

25 A.M. Dyugaev, Sov. Sci. Rev. A. Phys. 14, 1 (1990).

26 A. F. Andreev, JETP Lett. 28, 556 (1978) [Pisma v ZhETF 28, 603 (1978)]. 